\title{
Percepciones de alumnado y profesorado en Educación Superior sobre la evaluación en formacióninicial en educación física \\ Perceptions of students and tutors in Higher Education about assessment in teaching training in physical education

\author{
Luis Fernando Martínez Muñoz, María Luisa Santos Pastor, Francisco Javier Castejón Oliva \\ Universidad Autónoma de Madrid (España)
}

Resumen. La evaluación se constituye como un eje fundamental para lograr aprendizajes valiosos en la Educación Superior. Las percepciones del profesorado universitario respecto al alumnado en cuanto a la posibilidad de aplicar la evaluación formativa, no son coincidentes, lo que supone formas diferentes de proceder, y con ello, la dificultad de conseguir los resultados de aprendizaje previstos. El objetivo del estudio ha sido comprobar las diferentes percepciones sobre evaluación que tienen profesorado y alumnado, que afectan desde el diseño del programa hasta la relación entre la evaluación y la calificación. La población del estudio ha estado formada por 1133 estudiantes del Grado Maestro itinerario de Educación Física y del Grado de Ciencias de la Actividad Física y del Deporte, pertenecientes a 16 universidades españolas y a 217 profesores (63.1\% hombres y 36.9\% mujeres) de las mismas universidades y titulaciones. A ambas poblaciones se les pasó un cuestionario y posteriormente se hicieron dos grupos de discusión, uno de profesorado y otro de alumnado, de tres de las universidades del estudio. Las principales conclusiones muestran la poca coincidencia entre profesorado y estudiantes sobre la función, diseño y puesta en práctica de la evaluación. Esta circunstancia nos advierte sobre la necesidad de mejorar las prácticas de evaluación en la enseñanza universitaria, buscando una evaluación formativa orientada al aprendizaje.

Palabras claves: evaluación formativa, profesorado universitario, formación inicial, percepciones de los estudiantes, educación física

\begin{abstract}
Assessment is widely regarded as one of the essential factors that contribute to achieving valuable learning in Higher Education. University professors' perceptions of assessment do not always coincide with those of their students. Consequently, achieving the expected learning goals becomes more difficult. The aim of this study was to determine differences in the perception of assessment between teachers and students, from designing a program to the relation between evaluation and final grades. The population for this study comprised 1,133 students (68.4\% men and 31.6\% women) enrolled in Physical Education Teacher Education and in Physical Activity and Sports Science degrees of 16 Spanish universities, as well as 217 teachers (63.1\% men and 36.9\% women) teaching in the same degrees. Both students and teachers completed a questionnaire on their perceptions about assessment design. In addition, two discussion groups, for teachers and students from three of the institutions included respectively, were created. The main conclusions of the research highlight the lack of agreement between students and teachers on purpose, design and delivery of assessments. This suggests that there is a need to improve assessment practices in Higher Education, by implementing educational assessment focused on learning.
\end{abstract}

Keyword: educational assessment; university professors, teachers training, students’ perceptions, physical education.

\section{Introducción}

La evaluación se integra como un elemento más en el proceso de enseñanza aprendizaje (Knight, 2005). Debe orientarse al aprendizaje más que a la rendición de cuentas si se pretende que tenga la base formativa (Brown \& Glasner, 2007; Ramsden, 2003); lo que requiere definir con claridad las actividades para el docente y para el alumnado, evidencias, compromisos, etc., en suma, formas claras de entender y poner en práctica la evaluación (Bloxham \& Boyd, 2007; Yorke, 2003).

La mayoría de los estudios sobre la percepción de los estudiantes se centran en el punto de vista que tiene el profesorado sobre la evaluación, cómo la utilizan y la relación que tiene con los aprendizajes (Boussada \& De Ketele, 2008; Gijbels \& Dochy, 2006; Ibarra \& Rodríguez, 2014; Struyven, Dochy \& Janssens, 2005). Incluso algunos trabajos dirigen la atención hacia aspectos concretos de la evaluación (Gibbs \& Simpson, 2004-05; Hortigüela, Pérez \& Salicetti, 2015; Laight, Asghar \& AslettBentley, 2010). Lo que sí parece estar claro es que tanto el concepto de evaluación como su puesta en práctica, adquieren diferentes significados para el profesorado y el alumnado.

El principal interés del profesorado es utilizar la evaluación para que el alumnado consiga amplios y profundos aprendizajes (Yorke, 2003). En esta evaluación se debe prever cómo el alumnado tiene que conseguir los aprendizajes, con qué criterios se pueden comparar, así como quétipo de estrategias se deben seguir para fomentar la autonomía del alumnado (Hortigüela et al., 2015; Martos, Tamarit \& Torrent, 2016; Yorke, 2003). Es una evaluación en la que las intervenciones del alumnado cobran importancia durante las clases (Arribas, Manrique \& Tabernero, 2016; Nicol, 2010). Estas estrategias necesitan de instrumentos variados y un uso correcto del feedback que causen adecuados resultados del aprendizaje (Black \& Wiliam, 1998; Nicol, 2007; Nicol \&

Fecha recepción: 13-10-16. Fecha de aceptación: 19-12-16

F. Javier Castejón Oliva

javier.castejon@uam.es
Macfarlane-Dick, 2006; Sadler, 2010).

Cuando el diseño es el apropiado y las estrategias de evaluación se utilizan adecuadamente, la evaluación tiene potencial para ser formativa porque ayuda a mejorar los resultados de aprendizaje del alumnado. Es un diseño que tiene congruencia, desde los objetivos hasta la evaluación (Biggs, 2006; Biggs \& Tang, 2007; Carreiro, González \& González, 2016; Fernández \& Espada, 2017; López, Pérez, Manrique \& Monjas, 2016; Ramsden, 2003; Rushton, 2005). Si esta congruencia es precisa, los estudiantes perciben que están realizando los esfuerzos y aprendizajes adecuados (Bonsón \& Benito, 2007; Córdoba, 2015). Dunn y Mulvenon (2009), señalan que esta es la clave de una evaluación formativa, si bien los estudios no son concluyentes (por ejemplo, Bennett, 2011), de la misma forma que Yorke(2003) comenta la necesidad de una mejor fundamentación (ver también, López-Pastor, 2009).

Según cómo los estudiantes perciban el aprendizaje y la evaluación, así influirán en su motivación y rendimiento en las materias que estudian, reconduciendo las actividades hacia el aprendizaje activo y profundo, más que a la calificación (Gibbs, 2006; Gijbels, Segers \& Struyf, 2008; Romero, 2016; Trigueros, Rivera \& De la Torre, 2012). Es necesaria una evaluación que repercuta en su autonomía, más que en la memorización (Rué, 2009), y que el alumnado recibaun feedback lo más apropiado (Nicol, 2010).

Los estudios sobre la percepción de estudiantes y profesorado han sido realizados por grupos separados (docentes por un lado, estudiantes por otro) o por grupos confrontados (docentes y estudiantes). Se ha comprobado cómo percibe el alumnado la evaluación y los errores entendidos de la misma (Trillo \& Porto, 1999) o bien la coherencia entre la evaluación y el desarrollo de las competencias (Arribas et al., 2016). En cuanto a las diferencias en la percepción entre alumnado y profesorado (Gutiérrez, Pérez \& Pérez, 2013; Martínez, Castejón \& Santos, 2012; Ruiz, Ruiz \& Ureña, 2013), se observan que hay fallos en los planteamientos, sin duda sobre cómo se entiende todo el proceso. El profesorado manifiesta dominar el proceso, y señala que no puede ejercer por las propias dificultades que encuentra en su práctica (Pala- 
cios \& López-Pastor, 2013; Palacios, López \& Barba, 2013), sin embargo, el alumnado es consciente de lo que supone una buena evaluación y enseñanza (Gargallo, Sánchez, Ros \& Ferreras, 2010). Según estos estudios, todavía hay demanda sustancial para investigar las diferencias de apreciación entre alumnado y profesorado, y tratar de aportar más información para poder plantear soluciones, sobre todo cuando se trata de que el alumnado pueda participar en el proceso y si es posible, que lo que denominamos evaluación formativa y compartida, se cumpla.

El objetivo de este trabajo ha sido mostrar las diferencias de percepción que tiene el alumnado y el profesorado sobre la evaluación, desde el diseño de la evaluación en la guía docente, cómo se muestra y pone en práctica, las dificultades que se aprecia en el profesorado y cómo se relaciona la evaluación y la calificación.

\section{Metodología}

\section{Población}

Han participado 1133 estudiantes (775 hombres y 358 mujeres) que pertenecen a 16 universidades españolas que imparten el Grado de Maestro itinerario de EF y el Grado de Ciencias de la Actividad Física y el Deporte (Formación inicial del profesorado). Todos ellos asisten con asiduidad a sus clases. La media de edad de los participantes fue de 22 años. En cuanto al profesorado, participaron 217 profesores (137 hombres y 80 mujeres) de las mismas universidades y titulaciones que los estudiantes, con una edad media de 46 años, y una antigüedad en la docencia universitaria entre 5 y 21 años. Estos dos grupos cumplimentaron un cuestionario específico para cada población, tal y como se indica en el apartado de Instrumentos.

En los grupos de discusión han participado seis estudiantes, cuatro de último curso de Grado de Magisterio y dos de Ciencias de la Actividad Física, procedentes de tres universidades (dos de Madrid y la de Valladolid) donde se aplicaron los cuestionarios. Son estudiantes elegidos porque en el cuestionario aportaron información contradictoria en cuanto al tema tratado en dicho cuestionario, es decir, alumnado que tiene perspectivas enfrentadas. Para el profesorado se siguió el mismo proceso de procedencia (Madrid y Valladolid). Son nueve profesores, cinco de Magisterio y cuatro de Ciencias de la Actividad Física, con diversidad de experiencia docente (entre 5 y 18 años), y al igual que el alumnado, ofrecen respuestas enfrentadas.

\section{Instrumentos}

Para atender al objetivo de nuestra investigación, hemos utilizado dos instrumentos: el cuestionario y el grupo de discusión. El cuestionario es la «Escala sobremetodologías participativas y evaluación formativa en la formación inicial en educación física» (Castejón, Santos \& Palacios, 2015) que se diferencia entre el del profesorado y el del alumnado. Su enfoque es conocer las percepciones sobre la evaluación. Tiene un total de 38 preguntas que contienen las siguientes categorías: (a) presencia y comunicación de la evaluación en las programaciones; (b) coherencia entre los elementos del programa y la evaluación en las asignaturas; (c) puesta en práctica de la evaluación; y (d) la relación entre evaluación y calificación. Las valoraciones para cada una de las preguntas siguieron una escala Likert, con valores entre 0 y 4 , siendo 0 nada de acuerdo y 4 total acuerdo. La fiabilidad obtenida mediante los coeficientes alfa de Cronbach calculados para cada una de las escalas utilizadas resultaron satisfactorios (para los estudiantes: 38 preguntas, el alfa de Cronbach fue .866; para los profesores: 38 preguntas, el alfa de Cronbach fue 821).

En el grupo de discusión se utilizaron preguntas que procedían del cuestionario relacionadas con el objetivo de nuestra investigación. Se solicitaba a los miembros de cada grupo que expusieran sus pareceres sin que ahora tuvieran la limitación del cuestionario. Además, en cada grupo se solicitaba que dieran soluciones a partir de sus consideraciones sobre la evaluación.

Procedimiento

Los cuestionarios se pasaron durante el periodo escolar 2014-15, tras solicitar los respectivos permisos, indicando su carácter voluntario y el compromiso de confidencialidad de los datos obtenidos. Al alumnado se les administró en la última semana de clase, mientras que al profesorado en la semana siguiente a la finalización de las clases. En ambos casos, se guarda la confidencialidad por el grupo de investigación, de manera que solo los investigadores conocen a las personas que contestan a los cuestionarios.

Para los grupos de discusión se solicitó la participación del alumnado y del profesorado que atendía a nuestros objetivos del estudio, es decir, fueron elegidos por los investigadores según habían contestado al cuestionario. El desarrollo de ambos grupos se llevó cabo al final del periodo escolar, una vez analizados los cuestionarios. Se les informó del objetivo de la investigación y se les explicó el procedimiento. En cada grupo había dos personas del equipo investigador: un moderador y un secretario que tomaron nota y grabaron las conversaciones. La duración de cada grupo fue de tres horas. La trascripción de los dos grupos se pasó al coordinador de la investigación y se trianguló con el profesorado implicado en la investigación.

\section{Resultados}

En primer lugar se exponen los resultados del cuestionario para cada apartado relacionado con el objetivo del estudio, y se contrasta con el grupo de discusión. Hemos aplicado la media, desviación y la diferenciademedias con latde Studenta los dos grupos. El nivel designificatividad estadística se observa en $p>.005$.

En los grupos de discusión se identifica a las personas mediante un acrónimo con el fin de mantener el anonimato. Para el alumnado se utiliza la clave Es y para profesorado Pr; a continuación, irá el número asignado a la persona de cada grupo; Grado, MEF si es Magisterio del itinerario de EF o CAF si es de Ciencias de laActividad Física. Es decir, Es1CAF es el estudiante número 1 del Grado de Ciencias de la Actividad Física; mientras que Pr1MEF corresponde con el profesor número 1 Grado de Magisterio itinerario de EF.

Presencia y comunicación de la evaluación en la guía docente

El punto de partida corresponde sobre cómo está presente la información de la evaluación en las guías docentes y si se señalan los aprendizajes que se van a conseguir. El profesorado muestra valores medios más altos que los del alumnado en las dos preguntas iniciales (Tabla 1). Los aprendizajes que se espera conseguir en las asignaturas (recordemos que a partir del EEES, en las guías docentes se apuesta por los resultados de aprendizaje) son más valorados por el profesorado que por el alumnado.

Tabla 1

Comparación de medias (M) y desviación (DS) con relación al uso de la evaluación y la

\begin{tabular}{lcccccc}
\hline & $\begin{array}{c}\text { Alumnado } \\
(\mathrm{N}=1133)\end{array}$ & $\begin{array}{c}\text { Profesorado } \\
(\mathrm{N}=217)\end{array}$ & & & \\
\cline { 2 - 7 } & $\mathrm{M}$ & $\mathrm{DS}$ & $\mathrm{M}$ & $\mathrm{DS}$ & $t$ & Sig. \\
\hline $\begin{array}{l}\text { ¿La evaluación se corresponde con lo } \\
\text { que se encuentra en el programa de la } \\
\text { asignatura? }\end{array}$ & 2.81 & 0.625 & 3.72 & 0.526 & $-20,123$ & 0.000 \\
$\begin{array}{l}\text { ¿La evaluacín proporciona información } \\
\text { sobre el aprendizaje a conseguir? }\end{array}$ & 1.84 & 0.853 & 2.96 & 0.800 & -18.496 & 0.000 \\
\hline
\end{tabular}

Sin embargo, cuando se traslada la pregunta a los grupos de discusión, encontramos diferentes posturas: aunque hay una demanda por la participación, no es así percibida por el alumnado:

Los estudiantes entenderían mejor la evaluación si participan. Yo estoy hablando de que el estudiante participe en la evaluación sobre el proceso de enseñanza aprendizaje, aunque no en la evaluación sobre el propio alumno (Pr1MEF)

En la guía, yo les planteo dos tipos de evaluación y ellos deciden, pero con el compromiso, y es verbal, es un compromiso entre profesor y alumno (Pr5MEF)

El alumnado reclama que tienen que participar en la evaluación, no solo como receptores de una propuesta que se ha diseñado sin tenerles en cuenta:

Yo creo que se nos tendría que hacer partícipes de la evaluación a nosotros (Es4MEF). 
Lo que está diciendo mi compañero [se refiere a Es4MEF] es que para aprender a evaluar debes participar en la evaluación, que seas capaz tú de evaluar tu asignatura y de tu actuación en la asignatura (Es5CAF).

Ante la posibilidad de que esa evaluación sea formativa, en el profesorado aparecen perspectivas diferentes sobre cómo entender la evaluación y cómo debe estar presente en las guías. No todo el profesorado está de acuerdo con que la evaluación formativa sea una actuación que deba generalizarse, por ejemplo, en este debate del profesorado a favor y en contra:

Yo no estoy muy de acuerdo contigo en que sea una cuestión del contenido de la asignatura. O sea, que por ejemplo tú hagas evaluación formativa en unas asignaturas y en Anatomía no. O sea, yo creo que el profesor debe hacer evaluación formativa (Pr3MEF). ...pero la evaluación es un instrumento, no es un objetivo en sí. La evaluación es un instrumento (...)en cualquier asignatura(Pr8CAF). ...claro, es un instrumento de mejora del mismo proceso (Pr3MEF). Claro, pero para mí yo no pretendo lo mismo con un alumno de primero que tiene que aprenderse la anatomía que con un alumno de tercero. No hay tiempo para los mínimos, mucho menos para hacer cosas más creativas. Es un poco esas limitaciones que (...) número de alumnos, recursos, ciento y pico alumnos por aula y... (Pr8CAF). Yo lo que he visto también... yo este año he tenido a gente de primero y de cuarto y pienso que esto de la evaluación formativa también, cuando estás en primero lo que te toca haciendo evaluación formativa está bien con meter a los alumnos en este proceso y cuando estás en cuarto, si han hecho más evaluación formativa, es un gustazo (Pr3MEF).

En la misma línea, el alumnado señala cómo debería presentarse esa evaluación. Reclaman participar en la evaluación desde el principio, y no limitar su tarea a entregar información y a ser valorada por el profesorado:

Porque nosotros estamos hablando de un mínimo porcentaje, dos asignaturas de la carrera en las que podemos consensuar la evaluación (Es2MEF).

Es en ese tipo de evaluaciones cuando se ve si tú aprendes. Y no dejarlo para el día del examen, que puedes haber tenido un día mal y sacar mala nota (Es6MEF).

Coherencia entre los elementos del programa y la evaluación en las asignaturas

En la Tabla 2 aparecen los resultados que relacionan la evaluación con el resto de elementos del programa, teniendo en cuenta que la legislación expone que el profesorado asume el papel de responsable de elaborar sus propios programas.

\begin{tabular}{|c|c|c|c|c|c|c|}
\hline \multirow[t]{2}{*}{$\begin{array}{l}\text { Coherencia entre estrategias de } \\
\text { evaluación y calificación y... }\end{array}$} & \multicolumn{2}{|c|}{$\begin{array}{l}\text { Estudiantes } \\
(\mathrm{N}=1133)\end{array}$} & \multicolumn{2}{|c|}{$\begin{array}{c}\text { Profesorado } \\
(\mathrm{N}=217)\end{array}$} & \multirow[b]{2}{*}{$t$} & \multirow{3}{*}{ Sig. } \\
\hline & $\mathrm{M}$ & DS & M & DS & & \\
\hline Objetivos & 2.36 & 0.685 & 3.15 & 0.613 & -16.968 & \\
\hline Contenidos & 2.66 & 0.720 & 3.24 & 0.608 & -12.388 & 0.000 \\
\hline Competencias & 2.37 & 0.793 & 2.97 & 0.734 & -10.830 & 0.000 \\
\hline Metodología & 2.42 & 0.827 & 3.26 & 0.700 & -15.634 & 0.000 \\
\hline Evaluación y calificación & 2.59 & 0.802 & 3.23 & 0.656 & -12.307 & 0.000 \\
\hline
\end{tabular}

El profesorado tiene valores medios más altos que el alumnado en cada uno de los elementos señalados, si bien, podríamos decir que las competencias son las que aparecen menos valoradas, como tenemos que resaltar respecto al alumnado. Cuando preguntamos al profesorado sobre este aspecto, argumentan que hay una relación directa entre la forma de enseñar y de evaluar. Hay una fuerte crítica hacia el examen:

Darle un poco de coherencia entre qué y cómo enseñas al qué y cómo evalúas ¿̇no? (Pr7MEF).

Lo que creo es que no deberíamos ser es incoherentes. Hacemos evaluación formativa pero el mayor peso cae en el examen que vale más del cincuenta por ciento (Pr1MEF).

Desde la perspectiva del alumnado, es crítico cuando expone sus argumentos a la coherencia entre estos elementos curriculares, proponiendo una capacitación coherente con su desarrollo profesional:
Yo creo que es una evaluación formativa mal planteada. Está mal. Lo que tiene es el nombre simplemente. Porque si te dicen que va a ser formativa y luego el examen decide o condiciona tu nota, no es formativa (Es5CAF).

Con la evaluación habitual lo que aprendes es a coger rencor e intentar no hacerlo cuando tú salgas de la carrera. Porque yo eso lo tengo bien claro: yo eso no lo voy a intentar hacer (Es2MEF).

\section{Puesta en práctica de la evaluación}

A partir de los elementos curriculares y con el fin de dar respuesta a nuestro objetivo de investigación, exploramos cómo se pone en práctica la evaluación, las dificultades que se observan. Son los datos (Tabla 3) sobre las posibles causas o factores que alumnado y profesorado perciben para no poner en práctica una evaluación formativa según las condiciones que entiende cada grupo.

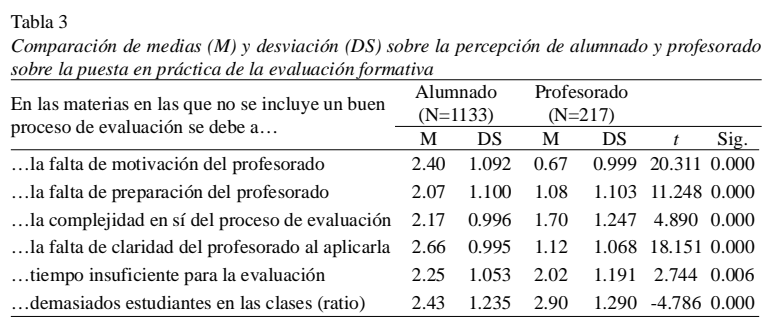

A diferencia de los datos anteriores, aquí se encuentran unos valores medios más bajos del profesorado que del alumnado. La explicación que encontramos es que el profesorado se apoya en razones estructurales (tiempo, ratio...) y no tanto en las personales. Sin embargo, el alumnado mantiene un equilibrio entre ambas razones. Esta divergencia nos hace comprender que hay diferencia de criterio entre lo que el alumnado y el profesorado valora sobre la evaluación.

Podríamos resumir que, al profesorado, desde su punto de vista, no le falta motivación ni conocimientos y oferta una evaluación clara, y que las dificultades las dirige hacia la ratio o a la escasez de tiempo; mientras que el alumnado percibe que es todo ese conjunto de factores de la tabla. Estas afirmaciones pueden verse corroboradas con lo que preguntamos al alumnado y profesorado en los grupos de discusión. El profesorado ratifica que el tiempo es insuficiente o que la ratio es elevada, lo que repercute en la adecuada revisión de las actividades para poder evaluarlas. Ambos grupos, docentes y discentes, aportan como solución una buena coordinación entre el profesorado:

Lógicamente es una cuestión de coordinación en cuanto a nosotros

[se refiere al profesorado]: de cómo queremos hacer la evaluación (Pr4CAF).

Si nos coordinamos con otros profesores para saber qué se está haciendo y qué no se está haciendo e incluso somos capaces de proponer actividades de evaluación que valgan para una asignatura y para otra, pero es una quimera (Pr1MEF).

En un instituto, cuando había un problema de cómo llevar a cabo entre todos evaluación formativa, conseguimos todo el profesorado hacer un criterio común. Aquí nos agobiamos, como decía la compañera, pensando hasta qué punto nosotros les mandamos muchísimos trabajos (Pr7MEF).

...el otro día comentábamos que menos mal que no todo el profesorado hacemos evaluación formativa. Tú fíjate que contra sentido ¿no? [se refiere a la carga de trabajo para el alumnado] (Pr5MEF).

En cuanto a los factores personales se comprueba entre alumnado y profesorado diferentes apreciaciones, y con ello, dificultades para entender los procesos y conseguir que la evaluación sea considerada como algo importante, no solo como un trámite de pruebas y poner las calificaciones:

Creo que domino los pasos que hay que dar para la evaluación formativa, pero si tú tienes un grupo de veinte personas o tienes un grupo de doscientas personas, por mucho que quieras hacer evaluación formativa eso te supone una carga de trabajo que al final es que es imposible. (Pr3MEF). 
Sabes que hay alternativas a grupos numerosos, que hay procesos para hacer la evaluación formativa, yo lo he leído en libros y artículos. Pero en la práctica me resulta difícil (Pr4CAF).

El profesor de la Universidad que se preocupa por su docencia, que se autoevalúa y que hace evolucionar sus clases, trabaja el doble. Encuentras satisfacción, pero a un precio... (Pr2CAF).

Yo hago evaluación continua, me encuentro motivado y comparto con [nombra a un profesor] el número de alumnos, y la verdad es que condiciona muchísimo. Yo tengo cien alumnos, no sé si ciento diez, y he intentado hacer evaluación formativa y de verdad ha sido la verdadera locura (Pr9MEF).

Para ellos también, porque a veces había sensaciones de 'pero por qué en una asignatura tenemos que tener tanto trabajo si tenemos otras asignaturas, si no podemos dedicarnos tanto a esta' (Pr1MEF). El alumnado considera que la evaluación formativa supone más trabajo y reclaman la coordinación entre el profesorado, para evitar discrepancias en la evaluación según sea el profesor de que se trate. El alumnado apunta la falta de coordinación del profesorado, sin embargo, reclaman que ese mismo profesorado les insista en la necesidad del trabajo en grupo en las clases:

Te prometo que, casi todo el mundo, se queja de trabajar demasiado. Lo que yo digo, si los docentes no se quejan... (Es3MEF). Se vuelve todo más exigente. Pero tiene que ser un trabajo en equipo entre los profesores, y entre profesor y alumno para mejorar todo el proceso (Es5CAF).

Requiere una coordinación entre los profesores, cosa que no hay en la Facultad (Es2MEF).

\section{Relación entre evaluación y calificación}

En la evaluación formativa y compartida hay una clara predisposición por implicar al alumnado en los procesos de evaluación, más allá de que muestren sus conocimientos y sea el profesorado el que controla todo el proceso. Implicar al alumnado supone ceder desde la propuesta hasta la puesta en práctica, pasando por el diseño, para que el alumnado se implique, sea un agente activo. Alos dos grupos se les preguntó sobre estos aspectos con el fin de comprobar si verdaderamente es una evaluación compartida (Tabla 4).

\begin{tabular}{|c|c|c|c|c|c|c|}
\hline \multirow[t]{2}{*}{ ¿Cómo se lleva a cabo la calificación? } & \multicolumn{2}{|c|}{$\begin{array}{c}\text { Alumnado } \\
(\mathrm{N}=1133)\end{array}$} & \multicolumn{2}{|c|}{$\begin{array}{l}\text { Profesorado } \\
(\mathrm{N}=217)\end{array}$} & \multirow[b]{3}{*}{ 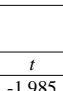 } & \multirow{3}{*}{$\begin{array}{l}\text { Sig. } \\
0.55\end{array}$} \\
\hline & $\mathrm{M}$ & DS & M & DS & & \\
\hline La calificación la decide el profesorado & 2.91 & 0.940 & 3.05 & 0.997 & & \\
\hline El alumno se autocalifica (parcial o totalm & 1.60 & 1.111 & 1.50 & 1.217 & 1.084 & 0.279 \\
\hline $\begin{array}{l}\text { Se califica de forma dialogada y consensuada (entre } \\
\text { profesorado y alumnado) (parcial o totalmente) }\end{array}$ & 1.53 & 1.188 & 1.54 & 1.210 & -0.202 & 0.840 \\
\hline $\begin{array}{l}\text { Se califica a partir de la autoevaluación (parcial o } \\
\text { totalmente) }\end{array}$ & 1.54 & 1.149 & 1.18 & 1.094 & 4.262 & 0.000 \\
\hline $\begin{array}{l}\text { Se califica a partir de la coevaluación (entre } \\
\text { compañeros) (parcial o totalmente) }\end{array}$ & 1.47 & 1.181 & 1.13 & 1.103 & 4.114 & 0.000 \\
\hline
\end{tabular}

Sigue siendo una valoración mayoritaria para ambos grupos que el profesorado es el que decide la calificación (primer ítem de la tabla), mientras que el resto de valores medios son bastante más bajos en ambos grupos. Comprobamos que los procesos de auto-evaluación y auto-calificación, calificación dialogada y coevaluación se valoran muy bajo por los dos grupos. Esto nos hace ver que no hay un sentido de implicación, bien sea por el profesorado a la hora de ofrecer esa posibilidad, bien por el alumnado a la hora de asumirla.

Estas diferencias se observan en los grupos de discusión. El alumnado tiene una clara aversión al examen y cómo se plantea; el profesorado remarca las diferencias entre evaluación formativa y cómo permite calificar al alumnado. Esta secuencia invita a entender cómo es el proceso y cómo se matiza la relación entre evaluación y calificación

Yo soy partidario de la evaluación formativa. Al final el alumno tiene un conocimiento claro desde el principio sobre su papel y la evaluación que se va a utilizar para calificar todo lo que él ha realizado durante el curso (Pr5MEF).

... la evaluación yo creo que debe ser coherente con la metodología que utilizamos en nuestras clases, tiene que permitir que el alumno no solamente reproduzca, sino que genere (Pr6CAF)
Se indica que tal cómo se plantean la evaluación y la calificación no siempre conduce a conductas responsables y el alumnado es crítico ante los planteamientos:

Lohevisto claramenteconuna pequeña experienciade autoevaluación que he tenido este año con alumnos de $4^{\circ}$ de educación física. Asílo he visto. No les importa incluso cambiar los criterios en el mismo instante, con tal de aprobar y tener razón (Pr4CAF).

Luego los alumnos finalizan su plan de estudios correspondientes y te dicen que están preparando oposiciones, y te comentan que la mayor parte de las asignaturas que ha cursado no le sirven para gran cosa (Pr9MEF).

...lo que vivo en mi formación es que se evalúa con exámenes que tienen la mayoría del peso en la calificación. Que se podían tener en cuenta otros aspectos como trabajos (Es5MEF).

Yo estoy de acuerdo con que dependiendo del instrumento que utilices, evalúa o no evalúa. Al final, lo que se suele hacer es un examen final, que no evalúa, califica (Es2MEF).

Las diferencias de apreciación percibidas, tanto por el profesorado como por el alumnado, muestran que no queda claro el rol que cada uno asume, tanto para el diseño de la evaluación y su puesta en práctica, como la transferencia desde la evaluación hasta la calificación.

\section{Discusión}

En nuestro estudio hemos comprobado que hay evidentes diferencias entre el alumnado y el profesorado sobre la forma de concebir la evaluación. Los resultados obtenidos, mediante cuestionarios, nos dicen que hay puntos de vista claramente diferentes. Los grupos de discusión nos ayudan a profundizar sobre aspectos relevantes referidos al proceso de la evaluación (su práctica y cómo mejorarla), en particular la evaluación formativa.

En el primer apartado referido a la evaluación que se concreta en las guías docentes o programaciones, hemos recogido que el alumnado tiene una percepción media bastante diferente respecto del profesorado (casi un punto entre medias), y es semejante a los que otros estudios han obtenido, como es el caso de Gutiérrez, Pérez, Pérez y Palacios (2011), el de Gutiérrez et al. (2013) o el de Gargallo et al. (2010), que destacan que un tercio del alumnado percibe que la evaluación se contempla convenientemente en las programaciones. Y lo mismo podemos decir respecto a los aprendizajes esperados, que nuestro estudio ofrece diferencias también de un punto entre las medias, similar a lo que muestran Gapp y Fisher (2006). Si deseamos una evaluación auténtica, que tenga el rasgo de evaluación formativa, tenemos que intentar que haya una implicación del alumnado porque favorece un ambiente de aprendizaje positivo (Struyven et al., 2005). En nuestro trabajo se percibe que el profesorado favorable a la evaluación formativa apuesta por una mayor implicación del alumnado, aunque también se detectan resistencias a ciertos procesos. Por otro lado, el alumnado estima que esa implicación repercute en una mejor formación (ver, por ejemplo, Brown \& Glasner, 2007; Ibarra \& Rodríguez, 2014; Martínez et al., 2012; Ruiz et al., 2013; Trillo \& Porto, 1999), y según nuestro estudio, añadimos que también en su desarrollo profesional.

La percepción que tiene el profesorado respecto a cómo existe una relación adecuada desde los objetivos hasta la evaluación, pasando por competencias, metodología y contenidos, es importante una coherencia entre todos los apartados, según la enseñanza alineada que propone Biggs (2006, ver también, Biggs \& Tang, 2007). El profesorado ofrece valores medios altos y ligeramente superiores a los que expone el alumnado, como no podría ser de otra forma pues así entienden su responsabilidad a la hora de diseñar las enseñanzas en las asignaturas. El alumnado valora menos esta coherencia, similar a los datos que han mostrado otros estudios (Arribas et al., 2016; Gutiérrez et al., 2011). Comprobamos en nuestros resultados que el profesorado percibe ser explícito en su programa, mientras que el alumnado no. En suma, hay que ser taxativos a la hora de presentar qué es lo que se espera del alumnado en la puesta en práctica de la evaluación, tal y como se reclama en diferentes estudios de investigación (Padilla \& Gil, 2008; Pérez et al., 
2008).

En cuanto a la percepción sobre la puesta en práctica de la evaluación, se aprecian diferencias para lograr que la evaluación suponga una buena práctica. Según nuestros resultados, mientras el profesorado achaca las dificultades para aplicar el programa a una cuestión estructural, fundamentalmente al tiempo y la ratio, el alumnado lo expresa como falta de dominio de la evaluación formativa, factores propios del profesorado, como la coordinación entre docentes o la falta de claridad al aplicarla, en la línea de los trabajos que ha mostrado estas incoherencias (Palacios \& López-Pastor, 2013; Palacios et al., 2013). El profesorado parece decir una cosa, lo que considera pedagógicamente correcto (trabajar en equipo), pero luego no siempre pone en práctica eso mismo que instruye (no se coordina). En el grupo de discusión, el alumnado solicita esa puesta en práctica de evaluación formativa, aunque haya dificultades, pues también la señalan como una evaluación más auténtica para conseguir los aprendizajes, similar al resultado de Hortigüela et al. (2015).

La evaluación compartida es una muestra de la mejora en la gestión de la clase y en concreto en la evaluación, tal y como hemos expuesto en el marco teórico; sin embargo, la percepción que tienen ambos grupos en nuestro estudio difiere claramente. El profesorado y el alumnado señalan claramente que son los docentes los que se encargan de la calificación con valores medios muy semejantes, datos que coinciden con los de Struyven etal. (2005), cuando el alumnado entiende que la evaluación se percibe como una imposición, o como ya señaló Carless (2006) el alumnado siente que se hace una valoración de medias para la calificación, pero no hay una calificación para la mejora del aprendizaje. Es en las diferentes estrategias de evaluación y su repercusión en la evaluación donde hemos obtenido valores medios bajos o muy bajos en ambos grupos. Según los trabajos que hemos comprobado, hay una apuesta fuerte por la evaluación compartida, por la evaluación entre iguales, por la autoevaluación y sus correspondientes relaciones con la calificación, y esos trabajos muestran repercusiones positivas en el aprendizaje (por ejemplo, Boud, 1989; Dochy, Segers \& Sluijsman, 1999; Falchikov \& Boud, 1989; Falchikov \& Goldfinch, 2000; Ibarra, Rodríguez \& Gómez, 2012; López-Pastor, Fernández, Santos \& Fraile, 2012; Rodríguez, Ibarra \& García, 2013). Son datos similares a los encontrados por Gutiérrez et al. (2011) cuando señalan que apenas se llevan a la práctica estrategias de evaluación compartida. Entendemos que no es fácil poner en práctica estos planteamientos, pues es necesario que el mismo profesorado universitario muestre un dominio y competencias de esa forma de proceder, que como ya hemos señalado en el apartado anterior, el alumnado no lo percibe así. Poner en práctica una evaluación formativa y compartida mejora los aprendizajes, aunque no está exenta de dificultades (Black \& Wiliam, 2009; Brookhart, 2007; Brown \& Glasner, 2007; López-Pastor, 2009).

\section{Conclusiones}

Las diferentes percepciones sobre la evaluación que tienen el alumnado y profesorado no son solo de terminología, también se refieren a la puesta en práctica y su consecuencia para la calificación, lo que nos lleva a entender las dos partes que participan en el proceso evaluativo. La falta de entendimiento, entre el profesorado y el alumnado, no es solo una cuestión de pareceres, es una falta de compromiso de ambas partes para que se consigan los aprendizajes y competencias necesarias en la formación inicial del profesorado.

Los objetivos de nuestra investigación son comprobar cómo perciben alumnado y profesorado la presencia del diseño de la evaluación en las guías docentes y su puesta en práctica y cómo se hace partícipe al alumnado en ese diseño. Además de cómo se entiende, si es o no formativa y cómo se materializan las estrategias de evaluación en la calificación, precisamente por la variedad que se solicita en esa evaluación formativa. Creemos que son objetivos importantes de investigación, porque estimamos que las diferencias entre estos grupos impiden un correcto desarrollo del proceso de enseñanza aprendizaje.

Hemos comprobado que el profesorado diseña la evaluación de la forma que la considera correcta pero el alumnado ha mostrado desacuerdos al respecto. Por supuesto, apenas hay negociación entre los dos grupos para la programación. A esto tenemos que añadir que la legislación universitaria española exige que las guías docentes estén publicadas antes del comienzo del curso, impidiendo, a nuestro parecer, que se pudiera realizar una verdadera negociación e implicar al alumnado en el propio diseño; si bien el profesorado tiene una responsabilidad en el diseño, sin embargo, si se hiciera partícipe al alumnado mejoraría la comprensión de estos documentos. En líneas generales, el profesorado señala que es congruente con lo que él ha expuesto en la documentación y así también es percibido por el alumnado. Pero, un alto porcentaje de ese profesorado señala que la evaluación debe informar de los aprendizajes del alumnado, aunque éstos manifiestan el escaso valor que tiene estainformación.

La coherencia que se comprueba entre los elementos de las guías docentes, desde los objetivos hasta los resultados, son asumidas por el profesorado como adecuadas, por lo que se advierte cierta coherencia, aunque el alumnado no lo percibe así. Los alumnos reclaman que la evaluación, debe ser formativa y estar bien planteada, pero no la realizan así, puesto que al final, la alineación desde los objetivos a la calificación, se resuelve en los exámenes. Los alumnos han manifestado que es así como les enseñan, pero no piensan hacerlo en su desarrollo profesional.

Por su parte, el profesorado percibe que desarrolla la evaluación bien, incluso que lo podría hacer mejor, si tuviera tiempo y una ratio adecuada. Sin embargo, el alumnado valora a la baja cualquier planteamiento propuesto por el profesorado. Este es un punto importante, porque las diferencias son las más acusadas. Si el profesorado, que es el que de partida tiene más responsabilidad, no accede a planteamientos compartidos, el alumnado no puede tomar partido y lo achaca a que el profesorado, en su conjunto, no quiere, más que no puede. En ambos grupos hemos detectado que una menor carga de trabajo, tanto para alumnado como para profesorado, y también una mejor coordinación entre el profesorado reduciría estas dificultades.

Por último, en la relación entre evaluación y calificación, parece que se asume la preponderancia del profesorado, quien determina la evaluación y calificación (y se relaciona con el primer objetivo del estudio). A partir de ahí, las diferentes formas de calificación no son bien valoradas por los dos grupos, esto indica que queda camino por recorrer para que este apartado pueda ser mejor investigado y que, verdaderamente suponga un cambio hacia una evaluación compartida entre los agentes que hemos estudiado: profesorado y alumnado. Es deseable que se eliminen las diferencias a la hora de entender la evaluación, y con ello, su puesta en práctica como determinante de los resultados de aprendizaje.

Las limitaciones de nuestro estudio se encuentran, fundamentalmente, en que hemos comprobado lo que dicen los agentes, pero no hemos analizado las guías docentes, que pueden ayudar a comprender mejor lo expuesto. Los grupos de discusión, por su propia naturaleza, limitan la representatividad, aunque sin duda, mejoran la profundidady calidad de la información. Una mayor presencia de otras universidades que no han participado en los grupos, aseguraría una mejora en los resultados de esta investigación. En el futuro, habría que ampliar este trabajo a otras titulaciones universitarias con el fin de tener una imagen más precisa sobre cómo se diseña y pone en práctica la evaluación y cómo es percibida por alumnado y profesorado en la universidad española y poder hacer estudios comparativos con universidades españolas y extranjeras.

\section{Agradecimientos}

Este artículo recoge datos del proyecto de investigación: EDU 2013-42024-R., del Programa Estatal de Investigación, Desarrollo e Innovación Orientada a los Retos de la Sociedad, en el marco del Plan Estatal de Investigación Científica y Técnica y de Innovación 20132016, denominado «Las competencias docentes en la formación inicial del profesorado de educación física». 


\section{Referencias}

Arribas, J.M., Manrique, J.C., \& Tabernero, B. (2016). Instrumentos de evaluación utilizados en la formación inicial del profesorado y su coherencia para el desarrollo de competencias profesionales en los estudiantes: visión del alumnado, egresados y profesorado. Revista Complutense de Educación, 27(1), 237-255.

Bennett, R.E. (2011). Formative assessment: a critical review. Assessment in Education: Principles, Policy, \& Practice, 18(1), 5-25. doi: 10.1080/0969594X.2010.513678

Biggs, J. (2006). Calidad del aprendizaje universitario. Madrid: Narcea.

Biggs, J., \& Tang, C. (2007). Teaching for Quality Learning at University. What the Student Does (3 ed.). Berkshire: McGraw-Hill. SRHE and Open University Press.

Black, P., \& Wiliam, D. (1998). Assessment and classroom learning. Assessment in Education: Principles, Policy \& Practice, 5(1), 7-74. doi: 10.1080 0969595980050102

Black, P., \& Wiliam, D. (2009). Developing the theory of formative assessment. Educationa Assessment, Evaluation, and Accountability, 21(1), 5-31. doi: 10.1007/s11092008-9068-5

Bloxham, S., \& Boyd, P. (2007). Developing Effective Assessment in Higher EducationA Practical Guide. Maidenhead: McGraw-Hill.

Bonsón, M., \& Benito, A. (2007). Evaluación y aprendizaje. En A. Benito \& A. Cruz (Coords). Nuevas claves para la docencia universitaria en el Espacio Europeo de Educación Superior (pp. 87-100). Madrid: Narcea.

Boud, D.J. (1989) The role of self assessment in formal grading procedures, Assessment and Evaluation in Higher Education, 14(1), 20-30. doi: 10.1080/ 0260293890140103

Boussada, H., \& De Ketele, J. M. (2008). L'évaluation de la qualité de la formation et du système d'évaluation universitaire: Le point de vue des diplômés. Avaliação, 13(1), 39-61. doi: 10.1590/s1414-40772008000100003

Brookhart, S. M. (2007). Expanding views about formative classroom assessment: A review of the literature. En J.H. McMillan (ed.), Formative classroom assessment Research, theory and practice (pp. 43-62). New York: Teachers College Press.

Brown, S., \& Glasner, A. (Eds.), (2007). Evaluar en la Universidad. Problemas y nuevos enfoques (2 ed.). Madrid: Narcea.

Carless, D. (2006). Differing perceptions in the feedback process. Studies in Higher Education, 31(2), 219-233, doi: 10.1080/03075070600572132

Carreiro, F., González, M.A., \& González, M.F. (2016). Innovación en la formación del profesorado de educación física. Retos, 29, 251-257.

Castejón, F. J., Santos, M. L., \& Palacios, A. (2015). Escala sobre metodologías participativas y evaluación formativa en la formación inicial en educación física. Revista Internacional de Medicina y Ciencias de la Actividad Física y el Deporte, 15(58), 245-267. doi: 10.15366/rimcafd2015.58.004

Córdoba, T. (2015). La aventura de aprender: Relato autobiográfico del viaje a Ítaca de un docente reflexivo. Retos, 28, 285-290.

Dochy, F., Segers, M., \& Sluijsman, D. (1999). The Use of Self-, Peer and Co-assessment in Higher Education: a review. Studies in Higher Education, 24(3), 331-350. doi:10.1080/03075079912331379935

Dunn, K. E., \& Mulvenon, S. W. (2009). A critical review of Research on Formative Assessments: The limited scientific evidence of the impact of Formative Assessments in education. Practical Assessment Research \& Evaluation, 14(7), 1-11.

Falchikov, N., \& Boud, D. (1989). Student Self-Assessment in Higher Education: A Meta-Analysis. Review of Educational Research, 59(4), 395-430. doi:10.3102 00346543059004395

Falchikov, N., \& Goldfinch, J. (2000). Student Peer Assessment in Higher Education: A Meta-Analysis Comparing Peer and Teacher Marks. Review of Educational Research, 70(3), 287-322. doi:10.3102/00346543070003287

Fernández, M, \& Espada, M.(2017). Formación inicial y percepción del profesorado sobre los estilos de enseñanza en Educación Física. Retos, 31, 69-75.

Gapp, R., \& Fisher, R. (2006). Achieving excellence through innovative approaches to student involvement in course evaluation within the tertiary education sector. Quality Assurance in Education, 14(2), 156-166. doi: 10.1108/ 09684880610662033

Gargallo, B., Sánchez, F., Ros, C., \& Ferreras, A. (2010). Estilos docentes de los profesores universitarios. La percepción de los alumnos de los buenos profesores. Revista Iberoamericana de Educación [on line], 51(4). [recuperado de http:// www.rieoei.org/deloslectores/3236Lopez.pdf].

Gibbs, G. (2006). How assessment frames student learning. En C. Bryan \& K. Clegg (Eds) Innovative Assessment in Higher Education (pp. 23-36). London: Routledge.

Gibbs, G., \& Simpson, C. (2004-05). Conditions under which assessment supports students' learning. Learning and Teaching in Higher Education, 1, 3-31.

Gijbels, D., \& Dochy, F. (2006). Students' assessment preferences and approaches to learning: can formative assessment make a difference? Educational Studies, 32(4), 399-409, doi: 10.1080/03055690600850354

Gijbels, D., Segers, M., \& Struyf, E. (2008). Constructivist learning environments and the (im)possibility to change students' perceptions of assessment demands and approaches to learning. Instructional Science, 36, 431-443. Doi: 10.1007/s11251008-9064-7

Gutiérrez, C., Pérez, A., \& Pérez, M. (2013). Percepciones de profesores, alumnos y egresados sobre los sistemas de evaluación en estudios universitarios de formación del profesorado de Educación Física. Ágora para la Educación Física y el Deporte, 15(2), 130-151.

Gutiérrez, C., Pérez, A., Pérez, M., \& Palacios, A. (2011). Percepciones de profesores y alumnos sobre la enseñanza, evaluación y desarrollo de competencias en estudios universitarios de formación de profesorado. Cultura y Educación, 23(4), 499-514. Doi: $10.1174 / 113564011798392451$

Hortigüela, D., Pérez, Á., \& Salicetti, A. (2015). ¿Cómo percibe el alumnado universitario de educación física la evaluación recibida? Contraste de dos metodologías diferentes. Retos, 28, 66-70.

Ibarra, M.S., \& Rodríguez, G. (2014). Modalidades participativas de evaluación: Un análisis de la percepción del profesorado y de los estudiantes universitarios. Revista de Investigación Educativa, 32(2), 339-361. doi: 10.6018/rie.32.2.172941

Ibarra, M.S., Rodríguez, G, \& Gómez, M.A. (2012). La evaluación entre iguales: beneficios y estrategias para su práctica en la universidad. Revista de Educación, 359 206-232. doi: 10-4438/1988-592X-RE-2011-359-092

Knight, P. (2005). El profesorado de Educación Superior. Formación para la excelencia. Madrid: Narcea.

Laight, J., Asghar, M., \& Aslett-Bentley, A. (2010). Investigating Conceptions and Practice of Formative Assessment in Higher Education. Literacy Information and Computer Education Journal, 1(3), 192-199. doi: 10.20533/licej.2040.2589.2010.0027

López-Pastor, V. M. (ed.). (2009). Evaluación formativa y compartida en Educación Superior. Propuestas, técnicas, instrumentos y experiencias. Madrid: Narcea.

López-Pastor, V.M., Fernández, J.M., Santos, M.L., \& Fraile, A. (2012). Students’ selfgrading, professor's grading and negotiated final grading at three university programmes: analysis of reliability and grade difference ranges and tendencies. Assessment \& Evaluation in Higher Education, 37(4), 353-364. doi:10.1080/ 02602938.2010.545868

López, V., Pérez, D., Manrique, J.C., \& Monjas, R. (2016). Los retos de la Educación Física en el siglo XXI. Retos, 29, 182-187.

Martínez, L.F., Castejón, F.J., \& Santos, M. L. (2012). Diferentes percepciones sobre evaluación formativa entre profesorado y alumnado en formación inicial en educación física. Revista Electrónica Interuniversitaria de Formación del Profesorado, 15(4). Recuperado de http://www.aufop.com/aufop/uploaded files/articulos/ 1364431164.pdf

Martos, D., Tamarit, E., \& Torrent, G (2016). Negociando el currículum en educación física. Una propuesta práctica de cogestión. Retos, 29, 223-228.

Nicol, D. (2007). Formative assessment and self-regulated learning: A model. Studies in Higher Education, 31(2), 199-218. doi: 10.1080/03075070600572090

Nicol, D. (2010). From monologue to dialogue: improving written feedback processes in mass higher education. Assessment \& Evaluation in Higher Education, 35(5), 501-517. doi: 10.1080/02602931003786559

Nicol, D., \& Macfarlane-Dick, D. (2006). Formative assessment and self-regulated learning: A model and seven principles of good feedback practice. Studies in Higher Education, 31(2), 199-218. doi: 10.1080/03075070600572090

Padilla, M.T., \& Gil, J. (2008). La evaluación orientada al aprendizaje en Educación Superior: condiciones y estrategias para su aplicación en la docencia universitaria. Revista Española de Pedagogía, 241, 467-486.

Palacios, A., \& López-Pastor, V. (2013). Haz lo que yo digo pero no lo que yo hago: sistemas de evaluación del alumnado en la formación inicial del profesorado. Revista de Educación, 361, 279-305. doi: 10.4438/1988-592X-RE-2011-361-143

Palacios, A., López, V.M., \& Barba, J.J. (2013). Tipologías de profesorado universitario en función de la evaluación aplicada en los futuros docentes. Estudios sobre Educación, 24, 173-195.

Pérez, A., Tabernero, B., López, VM., Ureña, N., Ruiz, E., Capllonch, M., ... Castejón, F.J. (2008). Evaluación formativa y compartida en la docencia universitaria: La concreción de cuestiones/clave para su aplicación en el camino hacia el Espacio Europeo de Educación Superior (EEES). Revista de Educación, 347, 183184.

Ramsden, P. (2003). Learning to teach in Higher Education. London: Routledge.

Rodríguez, G., Ibarra, M.S., \& García, E. (2013). Autoevaluación, evaluación entre iguales y coevaluación: conceptualización y práctica en las universidades españolas. Revista de Investigación en Educación, 11(2), 198-210.

Romero, M.R. (2016). La autoevaluación en expresión corporal en formación inicial del profesorado de educación física: un ejemplo de buena práctica. Retos, 29, 236-241.

Rué, J. (2009). El aprendizaje autónomo en la educación superior. Madrid: Narcea.

Ruiz, J.R., Ruiz, E., \& Ureña, N. (2013). La evaluación en la formación inicial del profesorado: que creemos hacer y que perciben los alumnos. Cultura, Ciencia y Deporte $9(8), 17,29$.

Rushton, A. (2005). Formative assessment: a key to deep learning? Medical Teacher 27(6), 509-513. doi: 10.1080/01421590500129159

Sadler, D.R. (2010). Beyond feedback: Developing student capability in complex appraisal. Assessment \& Evaluation in Higher Education, 35(5), 535-550. doi: 10.1080/ 02602930903541015

Struyven, K., Dochy, F., \& Janssens, S. (2005). Students’ perceptions about evaluation and assessment in higher education: a review. Assessment \& Evaluation in Higher Education, 30(4), 325-341. doi: 10.1080/02602930500099102

Trigueros, C., Rivera, E. \& De la Torre, E. (2012). La evaluación en el aula universitaria: del examen tradicional a la autoevaluación / The assessment in the university classroom: from traditional review to self-assessment. Revista Internacional de Medicina y Ciencias de la Actividad Física y el Deporte, 12(47), 473-491. Recuperado de http://cdeporte.rediris.es/revista/revista47/artevaluacion303.htm

Trillo, F., \& Porto, M. (1999). La percepción de los estudiantes sobre su evaluación en la universidad. Un estudio en la Facultad de Ciencias de la Educación. Innovación Educativa, 9, 55-75.

Yorke, M. (2003). Formative assessment in higher education: Moves towards theory and the enhancement of pedagogic practice. Higher Education, 45(4), 477-501. doi: 10.1023/A:1023967026413. 